\title{
PERSPECTIVE
}

\section{Pulmonary fibrosis: cytokines in the balance}

\author{
R.K. Coker, G.J. Laurent
}

\section{Pulmonary fibrosis: cytokines in the balance. R.K. Coker, G.J. Laurent. @ERS Journals Ltd 1998.}

ABSTRACT: Pulmonary fibrosis can complicate diverse pulmonary and systemic pathologies. In many cases the underlying cause remains unidentified. Mortality from the disease is increasing steadily in the UK and USA. The clinical features are welldescribed, but patients frequently present at an advanced stage, and current treatments have not improved the poor prognosis. There is a compelling need to identify the fibrotic process earlier and to develop new therapeutic agents.

Increased collagen deposition is central to the pathology and interest over the last decade has focused on the role of cytokines in this process. These polypeptide mediators are believed to be released from both circulating inflammatory and resident lung cells in response to endothelial and epithelial injury. Key cytokines currently implicated in the fibrotic process are transforming growth factor- $\beta$, tumour necrosis factor- $\alpha$ and endothelin-1.

This article outlines the evidence implicating these mediators in the pathogenesis of pulmonary fibrosis and also considers the possible role of cytokines with antifibrotic effects, such as interferon- $\gamma$. The "balance" of positively and negatively regulating cytokines is discussed, and the potential for interaction with other factors including viruses, hormones and altered antioxidant status is also considered.

Finally, potential novel therapeutic approaches are discussed, together with suggestions for future studies and clinical trials. As the outcomes of different avenues of research over the last ten years are brought together, it is clear that there is now a hitherto unrivalled opportunity to begin to tackle the treatment of this devastating disease. Eur Respir J 1998; 11: 1218-1221.
Centre for Respiratory Research, University College London Medical School, London, UK.

Correspondence: R.K. Coker

Centre for Respiratory Research

University College London Medical School

Rayne Institute

5 University Street

London WC1E 6JJ

UK

Fax: 441712096973

Keywords: Cytokines

endothelin-1

fibrosis

lung

transforming growth factor- $\beta$

tumour necrosis factor- $\alpha$

Received: January 151998

Accepted for publication January 151998

\section{Clinical and biochemical features of cryptogenic fibrosing alveolitis}

Pulmonary fibrosis is the final common sequel to a variety of pathologies, which include lung injury resulting from dust inhalation, radiation or drugs, and systemic and pulmonary diseases. The latter encompass connective tissue disorders, sarcoidosis and infections, such as tuberculosis, mycoplasma and pneumocystis. Cryptogenic fibrosing alveolitis (CFA) (idiopathic pulmonary fibrosis (IPF) in the USA) describes pulmonary fibrosis arising in the absence of known predisposing factors.

In the UK, prevalence of CFA alone is about five per 100,000 , and annual mortality, currently around 2,000, has more than doubled since 1980. Prevalence in the USA is similar and mortality is also rising. Affected individuals typically present with nonproductive cough and breathlessness on exertion, which progresses at a variable rate. Clinical findings include finger clubbing, fine inspiratory crackles at the lung bases, and a restrictive defect on lung function testing with reduced gas transfer. Widening of the alveolaarterial (A-a) gradient on exercise may be the only abnormality early on in the course of the disease (see review [1]).

Patients tend to present late in the course of disease, and current treatment, comprising corticosteroids with or without immunosuppressants, has not improved the prognosis. Most studies show approximately 50\% 5-yr survival, irrespective of therapy [2]. The evidence thus indicates an urgent need to identify patients with the disease at an earlier stage, and to develop new forms of treatment, which could be used alone or in conjunction with conventional therapy.

The pathological features of CFA are well described (see review [3]) and comprise infiltration of inflammatory cells together with deposition of collagen. These changes result in interstitial thickening and loss of alveolar architecture, leading to reduced lung volume and impaired gas exchange. Fibroblast proliferation at sites of injury also contributes to collagen accumulation.

\section{Role of cytokines}

Over the last decade, considerable evidence has emerged suggesting that important stimuli to collagen deposition in pulmonary fibrosis are polypeptide mediators known as cytokines [4]. Released by resident lung cells and recruited inflammatory cells, cytokines are thought to stimulate fibroblast proliferation and increased synthesis of extracellular matrix proteins, including collagen.

An early feature in the pathogenesis of CFA is alveolar epithelial and/or capillary cell injury. This promotes recruitment into the lung of circulating immune cells, such as monocytes, neutrophils, lymphocytes and eosinophils. These effector cells, together with resident lung cells, such as macrophages, alveolar epithelial and endothelial cells, then release cytokines, which stimulate target cells, typi- 
cally fibroblasts, to replicate and synthesize increased amounts of collagen. Breakdown of extracellular matrix protein may also be inhibited, thereby contributing to the fibrotic process.

Numerous cytokines have been implicated in the pathogenesis of lung fibrosis, including transforming growth factor- $\beta$ (TGF- $\beta$ ), tumour necrosis factor- $\alpha$ (TNF- $\alpha$ ), platelet-derived growth factor (PDGF), insulin-like growth factor-1 (IGF-1), endothelin-1 (ET-1) and the interleukins, (IL)-1 and IL-8 [5]. Chemokine leukocyte chemoattractants, including the factor regulated upon activation in normal T-cells, expressed and secreted (RANTES), are also thought to play an important role [6]. This has led to speculation that anticytokine therapeutic strategies, designed to inhibit cytokine production or function, will represent a new and effective treatment for CFA. Before considering whether such treatment may have a role to play in the future, it is necessary to review the evidence implicating cytokines in the fibrotic process. Current data are most convincing for TGF- $\beta$, TNF- $\alpha$ and ET-1.

The TGF- $\beta$ family of proteins has the most potent stimulatory effect on extracellular matrix deposition of any cytokines so far examined. In vitro studies show that TGF$\beta_{1}$, secreted as a latent precursor, promotes fibroblast procollagen gene expression and protein synthesis. The data suggest that the other mammalian isoforms, TGF- $\beta_{2}$ and TGF- $\beta_{3}$, also stimulate human lung fibroblast collagen synthesis and reduce breakdown in vitro [7]. In animal models of pulmonary fibrosis, enhanced TGF- $\beta_{1}$ gene expression is temporally and spatially related to increased collagen gene expression and protein deposition [8]. TGF- $\beta_{1}$ antibodies reduce collagen deposition in murine bleomycininduced lung fibrosis [9], and human fibrotic lung tissue shows enhanced TGF- $\beta_{1}$ gene and protein expression [10].

TNF- $\alpha$ can stimulate fibroblast replication and collagen synthesis in vitro, and pulmonary TNF- $\alpha$ gene expression rises after administration of bleomycin in mice [11]. Soluble TNF- $\alpha$ receptors reduce lung fibrosis in murine models [12], and pulmonary overexpression of TNF- $\alpha$ in transgenic mice is characterized by lung fibrosis [13]. In patients with CFA or asbestosis, bronchoalveolar lavage fluid-derived macrophages release increased amounts of TNF- $\alpha$ compared with controls [14].

ET-1 also fulfils the criteria for a profibrotic cytokine. This molecule promotes fibroblast proliferation and chemotaxis [15] and stimulates procollagen production [16]. It is present in the lungs of patients with pulmonary fibrosis [17], and a recent report suggests that the ET-1 receptor antagonist, bosentan, ameliorates lung fibrosis when administered to experimental animals [18].

Taken together, these data suggest that anticytokine therapeutic approaches may be of value in treating patients with CFA. However, other aspects may have to be taken into consideration. First, not all cytokines appear to be profibrotic. In particular, evidence from animal and human studies suggests that interferon- $\gamma$ (IFN- $\gamma$ ) may have antifibrotic effects. IFN- $\gamma$ inhibits fibroblast collagen synthesis in vitro [19] and attenuates bleomycin-induced lung fibrosis when administered to mice [20]. Diminished IFN$\gamma$ reported in patients with CFA could, therefore, enhance collagen accumulation [21]. Figure 1 suggests how a "balance" of positively and negatively regulating cytokines might modulate the fibrotic response to injury.
Secondly, it is clear that cytokines can interact in vivo to produce different effects from those observed in vitro, and that the target cell phenotype can modulate the res-ponse to a variety of mediators. Genetic factors and the stage of disease may, therefore, prove to be a crucial element in determining whether cytokine-modifying therapy is effective in individual patients.

Thirdly, redundancy of cytokine action may prove to be an important feature in the pathogenesis of CFA, such that if the action of one particular profibrotic mediator is inhibited, that of another is automatically upregulated to take its place.

Fourthly, cytokines are not the only factors thought to be capable of initiating or modulating the fibrotic response to injury. Recent interest has focused on blood-derived proteins, including thrombin [22]; however, a variety of physical, chemical and hormonal agents, probably interacting with cytokines, are also recognized to modulate collagen synthesis [4]. Altered antioxidant status, with pulmonary glutathione deficiency, has also been proposed as a factor influencing the degree of lung injury and subsequent fibrosis in CFA, leading to studies of antioxidant therapy in the bleomycin model [23] and in patients [24]. A viral aetiology for CFA has also been proposed, one particular candidate being the Epstein-Barr virus (EBV). Raised serum antibodies to EBV and immunoglobulin A (IgA) against viral capsid antigen have been documented in 13 patients with CFA [25], whilst EGAN et al. [26] identified antigen specific for replicating EBV in alveolar type II cells in lung biopsies from patients with CFA. A recent study, however, failed to demonstrate either EBV ribonucleic acid (RNA) or EBV deoxyribonucleic acid (DNA) in

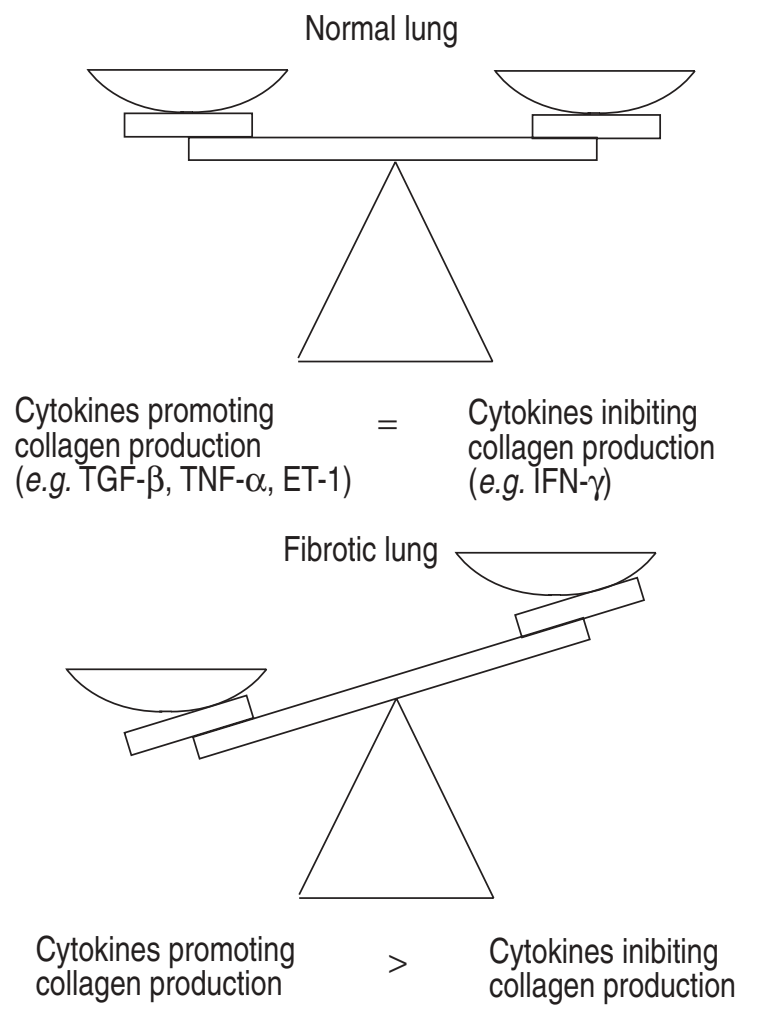

Fig. 1. - Cytokines in the balance in normal and fibrotic lung. TGF- $\beta$ : transforming growth factor- $\beta$; TNF- $\alpha$ : tumour necrosis factor- $\alpha$; ET- 1 : endothelin-1; IFN- $\gamma$ : interferon- $\gamma$. 
lung tissue from patients with CFA or fibrosing alveolitis associated with systemic sclerosis [27]. This area therefore remains controversial.

Fifthly, various cytokines may, in addition to direct effects on collagen metabolism, promote further pulmonary inflammation by upregulating expression of adhesion molecules and thereby recruitment of leukocytes [28]. In this context, it is interesting to note that administration of monoclonal antibodies to leukocyte integrins prevents lung collagen deposition in the bleomycin mouse model, even when administration is given at a late stage when collagen deposition is almost complete [29].

Finally, other factors besides IFN- $\gamma$ may serve to protect the normal lung against fibrosis. One example is that of prostaglandin $\mathrm{E}_{2}\left(\mathrm{PGE}_{2}\right)$. Recent data from our laboratory show that $\mathrm{PGE}_{2}$ plays a key role in mediating the antiproliferative effects of TGF- $\beta$ on human lung fibroblasts [30]. Other groups have previously suggested that fibroblasts from patients with pulmonary fibrosis have an impaired capacity to synthesize $\mathrm{PGE}_{2}[31,32]$. In the presence of increased $T G F \beta_{1}$ levels in the lung, $\mathrm{PGE}_{2}$ deficiency could thus lead to increased fibroblast proliferation and thereby enhance the fibrotic response.

\section{Implications for new treatments}

It is clear that novel strategies are required in the treatment of pulmonary fibrosis. Specific anticytokine therapies will require evaluation, used either singly or in combination. Anticytokine strategies will also need to be evaluated alone or together with conventional treatment, such as immunosuppressant agents. Agents could potentially be developed which inhibit the effects of several cytokines together, for example using transcription inhibitors. New classes of agents, such as the pyridinyl-imidazole compounds, which inhibit both IL-1 and TNF- $\alpha$ release from stimulated human monocytes [33], may have therapeutic potential. Figure 2 illustrates the different strategies which might be employed to inhibit the effects of profibrotic cytokines.

Agents which lead to upregulation of negatively acting cytokines, such as IFN- $\gamma$, may also have therapeutic potential and should be investigated. Therapeutic strategies to enhance $\mathrm{PGE}_{2}$ activity could also prove to be of value. Ultimately, a combination of agents acting via different mechanisms, used in combination either concurrently or sequentially, may be necessary.

Now is an attractive and, indeed, essential time to evaluate cytokine-based strategies in pulmonary fibrosis. The agents are now becoming available and the hypotheses put forward by basic science research need to be tested clinically. Somewhat disappointing results with antiTNF therapy in patients with acute lung injury should not necessarily deter investigators. Further research may, for example, lead to identification of subgroups of patients who are "high cytokine producers" and, therefore respond optimally to such treatments.

However, it is clear that further studies will be needed on other factors influencing the progression of disease. These should ideally include genetic studies as well as investigation into the mechanisms by which local lung environments influence cytokine action. Furthermore, new antifibrotic agents, such as pentoxifylline, a xanthine which indirectly inhibits TNF- $\alpha$ synthesis, probably by inhibiting phos-

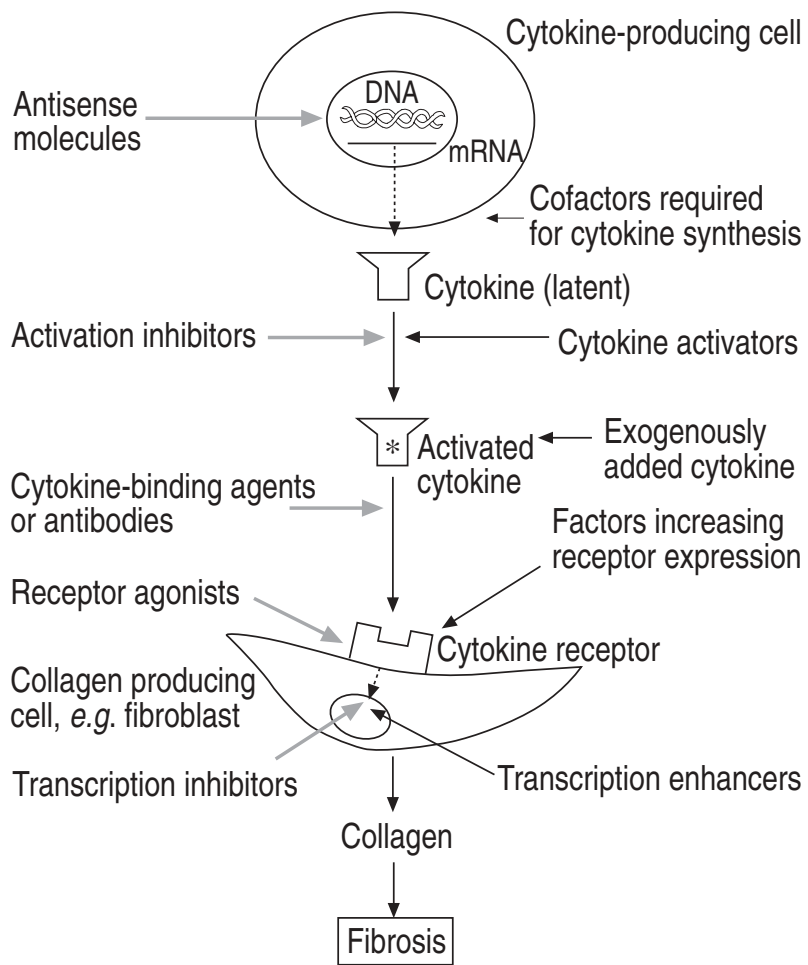

Fig. 2. - Potential use of cytokine inhibitors or activators as antifibrotic agents. $\longrightarrow$ : cytokine inhibitors; $\longrightarrow$ : cytokine activators. DNA: deoxyribonucleic acid; mRNA: messenger ribonucleic acid.

phodiesterase activity [34], and halofuginone, a specific, reversible inhibitor of type I collagen synthesis [35], are appearing as rivals in the therapeutic arena.

If new therapeutic strategies are to be evaluated effectively, there will be a need to assess markers of disease before, during and after completion of therapy. At present, these seem likely to include lung function testing, bronchoalveolar lavage levels of cytokines and procollagen peptides, high-resolution computed tomography (CT) scanning and measurement of lung epithelial permeability using diethylenetriamine penta-acetate (DTPA) scanning. The development of precise, safe and noninvasive assessments will be crucial to the success of such studies.

Finally, there is a need for increased clinical awareness of CFA and further epidemiological studies alongside basic laboratory studies. This will enable patients to be identified earlier in the course of the disease, when treatment is most likely to be effective, and to learn more about its natural course. Of particular interest may be patients with coexistent rheumatological and lung disease, or inflammatory bowel and lung disease. A significant proportion of these patients have a subclinical alveolitis. Studies in these patients may provide clues as to the factors determining progression or resolution, and the rates of recovery or decline.

In conclusion, over the last 10 yrs there have been great gains in our understanding of the mechanisms regulating collagen synthesis and degradation, including the role played by cytokines. The nature of cytokine genes, mechanisms of cytokine activation and their receptors is being unravelled, suggesting new therapeutic targets. Further epidemiological, genetic and laboratory studies are required to clarify the mechanisms responsible for initiating the fibrotic process. Current treatment is unsatisfactory and 
cytokine-modulating therapy could be an essential tool in treatment, perhaps in conjunction with other treatments. Understanding the balance of cytokines in health and disease will be crucial for the success of such therapy. However, other factors besides cytokines probably modulate the fibrotic process, and treatment directed at modifying the activity of these factors may also prove to be important. While the trials of cytokine-based therapy and other new antifibrotic agents are awaited, the role of cytokines remains in the balance. The next decade has the potential to be an exciting one in this field.

\section{References}

1. Turner-Warwick M, Burrows B, Johnson A. Cryptogenic fibrosing alveolitis: clinical features and their influence on survival. Thorax 1980; 35: 171-180.

2. Turner-Warwick M, Burrows B, Johnson A. Cryptogenic fibrosing alveolitis: response to corticosteroid treatment and its effect on survival. Thorax 1980; 35: 593-599.

3. Kuhn C. Pathology. In: Phan SH, Thrall RS, eds. Pulmonary Fibrosis. New York, Marcel Dekker, 1995; pp. 59-83.

4. McAnulty RJ, Laurent GJ. Collagen and its regulation. In: Phan SH, Thrall RS, eds. Pulmonary Fibrosis. New York, Marcel Dekker 1995; pp. 135-171.

5. Coker RK, Laurent GJ. Pathogenesis of pulmonary fibrosis: implications for pharmacological intervention. In: Walter EH, du Bois RM, eds. Immunology and Management of Interstitial Lung Disease. London, Chapman and Hall 1995; pp. 19-36.

6. Keane MP, Standiford TJ, Strieter RM. Chemokines are important cytokines in the pathogenesis of interstitial lung disease. Eur Respir J 1997; 10: 1199-1202.

7. Coker RK, Laurent GJ, Shahzeidi S, et al. Transforming growth factor $\beta_{1},-\beta_{2}$ and $-\beta_{3}$ all stimulate fibroblast procollagen production in vitro but are differentially expressed during bleomycin-induced lung fibrosis. $\mathrm{Am} \mathrm{J}$ Pathol 1997; 150: 981-991.

8. Hoyt DG, Lazo JS. Early increases in pulmonary mRNA encoding procollagens and transforming growth factor- $\beta$ precede bleomycin-induced pulmonary fibrosis in mice. $J$ Pharmacol Exp Ther 1988; 246: 765-771.

9. Giri SN, Hyde DM, Hollinger MA. Effect of antibody to transforming growth factor $\beta$ on bleomycin induced accumulation of lung collagen in mice. Thorax 1993; 48: 959-966.

10. Broekelmann TJ, Limper AH, Colby TV, McDonald JA. Transforming growth factor $\beta_{1}$ is present at sites of extracellular matrix expression in human pulmonary fibrosis. Proc Natl Acad Sci 1991; 88: 6642-6646.

11. Piguet PF, Collart MA, Grau GE, et al. TNF/cachectin plays a key role in bleomycin-induced pneumopathy and fibrosis. J Exp Med 1989; 170: 655-663.

12. Piguet PF, Vesin C. Treatment by human recombinant soluble TNF receptor of pulmonary fibrosis induced by bleomycin or silica in mice. Eur Respir J 1994; 7: 515-518.

13. Miyazaki Y, Araki K, Vesin C, et al. Expression of a tumor necrosis factor- $\alpha$ transgene in murine lung causes lymphocytic and fibrosing alveolitis. J Clin Invest 1995; 96: 250-259.

14. Zhang Y, Lee TC, Guillemin B, et al. Enhanced IL-1 $\beta$ and tumor necrosis factor $\alpha$ release and messenger RNA expression in macrophages from idiopathic pulmonary fibrosis or after asbestosis exposure. J Immunol 1993; 150: 4188-4196.

15. Peacock AJ, Dawes KE, Shock A, Gray AJ, Reeves JT, Laurent GJ. Endothelin 1 and endothelin 3 induce chemotaxis and replication of pulmonary artery fibroblasts. $A m$ Rev Respir Cell Mol Biol 1992; 7: 492-499.

16. Dawes KE, Cambrey AD, Campa JS, et al. Changes in collagen metabolism in response to endothelin-1: evi- dence for fibroblast heterogeneity. Int J Biochem Cell Biol 1996; 2: 229-238.

17. Cambrey A, Harrison NK, Dawes KE, et al. Increased levels of endothelin-1 in bronchoalveolar lavage fluid from patients with systemic sclerosis contribute to fibroblast mitogenic activity in vitro. Am J Respir Cell Mol Biol 1994; 11: 439-445.

18. Park S-H, Saleh D, Giaid A, Michel RP. Increased endothelin-1 in bleomycin-induced pulmonary fibrosis and the effect of an endothelin receptor antagonist. Am J Respir Crit Care Med 1997; 156: 600-608.

19. Rosenbloom J, Feldman G, Freundlich B, Jimenez SA. Transcriptional control of human diploid fibroblast collagen synthesis by $\gamma$-interferon. Biochem Biophys Res Commun 1984; 123: 365-372.

20. Hyde DM, Henderson TS, Giri SN, et al. Effect of murine-gamma interferon on the cellular responses to bleomycin in mice. Exp Lung Res 1988; 14: 686-704.

21. Prior C, Haslam P. In vivo levels and in vitro production of interferon gamma in fibrosing interstitial lung diseases. Clin Exp Immunol 1992; 88: 280-287.

22. Hernandez-Rodriguez NA, Cambrey AD, Harrison NK, et al. Role of thrombin in pulmonary fibrosis. Lancet 1995; 346: 1071-1073.

23. Shahzeidi S, Sarnstrand B, Jeffery PK, McAnulty RJ, Laurent GJ. Oral N-acetylcysteine reduces bleomycininduced collagen deposition in the lungs of mice. Eur Respir J 1991; 4: 845-852.

24. Meyer A, Buhl R, Magnussen H. The effect of oral $\mathrm{N}$-acetylysteine on lung glutathione levels in idiopathic pulmonary fibrosis. Eur Respir J 1994; 7: 431-436.

25. Vergnon JM, de Thé G, Weynants P, Vincent M, Mornex JF, Brune J. Cryptogenic fibrosing alveolitis and EpsteinBarr virus: an association. Lancet 1984; ii: 768-770.

26. Egan JJ, Stewart JP, Hasleton PS, Arrand JR, Carroll KB, Woodcock AA. Epstein-Barr virus replication within pulmonary epithelial cells in cryptogenic fibrosing alveolitis. Thorax 1995; 50: 1234-1239.

27. Wangoo A, Shaw RJ, Diss TC, Farrell PJ, du Bois RM, Nicholson AG. Cryptogenic fibrosing alveolitis: lack of association with Epstein-Barr virus infection. Thorax 1997; 52: 888-891.

28. Churchill L, Gundel RH, Letts LG, Wegner CD. Contribution of specific cell-adhesive glycoproteins to airway and alveolar inflammation and dysfunction. Am Rev Respir Dis 1993; 148: S83-S87.

29. Piguet PF, Rosen H, Vesin C, Grau GE. Effective treatment of the pulmonary fibrosis elicited in mice by bleomycin or silica with anti-CD-11 antibodies. Am Rev Respir Dis 1993; 147: 435-441.

30. McAnulty RJ, Hernandez-Rodriguez NA, Mutsaers SE, Coker RK, Laurent GJ. Indomethacin suppresses the antiproliferative effects of transforming growth factor- $\beta$ isoforms on fibroblast cell cultures. Biochem $J$ 1997; 321: 639-643.

31. Borok Z, Gillissen A, Buhl R, et al. Augmentation of functional prostaglandin $E$ levels on the respiratory epithelial surface by aerosol administration of prostaglandin E. Am Rev Respir Dis 1991; 144: 1080-1084.

32. Wilborn J, Crofford LJ, Burdick MD, Kunkel SL, Strieter RM, Peters-Golden M. Cultured lung fibroblasts isolated from patients with idiopathic pulmonary fibrosis have a diminished capacity to synthesise prostaglandin $\mathrm{E}_{2}$ and to express cyclooxygenase-2. J Clin Invest 1995; 95: 1861-1868.

33. Lee JC, Laydon JT, McDonnell PC, et al. A protein kinase involved in the regulation of inflammatory cytokine biosynthesis. Nature 1994; 372: 739-746.

34. Zabel P, Entzian P, Dalhoff K, Schlaak M. Pentoxifylline in treatment of sarcoidosis. Am J Respir Crit Care Med 1997; 155: 1665-1669.

35. Fishman RHB. Kosher chickens, rabbits' ears, and a scar-free future. Lancet 1997; 350: 570. 